\title{
SLIDING HUMP TECHNIQUE AND SPACES WITH THE WILANSKY PROPERTY
}

\author{
DOMINIKUS NOLL AND WOLFGANG STADLER
}

(Communicated by John B. Conway)

\begin{abstract}
We prove that if $E$ is a $B K-A K$-space whose dual $E^{\prime}$ as well is $B K-A K$, then $\sigma\left(E^{\prime}, F\right)$ and $\sigma\left(E^{\prime}, \bar{F}\right)$ have the same convergent sequences whenever $F$ is a subspace of $E^{\prime \prime}$ containing $\Phi$ and satisfying $F^{\beta}=E^{\beta}$. This extends a result due to Bennett $\left[\mathrm{B}_{2}\right]$ and the second author [S]. We provide new examples of $B K$-spaces having the Wilansky property. We show that the bidual $E^{\prime \prime}$ of a solid $B K-A K$-space $E$ whose dual as well is $B K-A K$ satisfies a separable version of the Wilansky property. This extends a theorem of Bennett and Kalton, who proved that $l^{\infty}$ has the separable Wilansky property.
\end{abstract}

\section{INTRODUCTION}

G. Bennett $\left[\mathrm{B}_{2}\right]$ and the second author $[\mathrm{S}]$ have independently obtained a positive answer to the following question of Wilansky: Is $c_{0}$ the only $F K$-space, densely containing $\Phi$, whose $\beta$-dual is $l^{1}$ ? Both approaches are essentially based on a characterization of the barrelledness of certain sequence spaces by means of their $\beta$-duals. In the present paper we extend the Bennett/Stadler result, providing more examples of $B K$-spaces having the Wilansky property (in the sense introduced in $\left[B_{2}\right]$ ).

Let us explain the situation by considering a typical example. The classical sliding hump argument (Toeplitz/Schur) asserts that $\sigma\left(l^{1}, c_{0}\right)$-bounded sets are \|\|$_{1}$-bounded. The Bennett/Stadler result generalizes this to the extent that still $\sigma\left(l^{1}, E\right)$-bounded sets are \|\|$_{1}$-bounded, when $\Phi \subset E \subset c_{0}$ and $E^{\beta}=l^{1}$. The latter may be expressed equivalently by saying that every subspace $E$ of $c_{0}$ containing $\Phi$ and having $E^{\beta}=l^{1}$ is barrelled. Finally, our present attempt shows that $\sigma\left(l^{1}, E\right)$-bounded sets are \|\|$_{1}$-bounded when $\Phi \subset E \subset l^{\infty}$ and $E^{\beta}=l^{1}$. Actually, we prove a little more. We show that $\sigma\left(l^{1}, E\right)$ and $\sigma\left(l^{1}, \bar{E}\right)$ have the same convergent sequences in case $\Phi \subset E \subset l^{\infty}$ and $E^{\beta}=l^{1}$. This extension requires a modified technique, since both the approaches in $\left[B_{2}\right]$ and

Received by the editors December 16, 1987.

1980 Mathematics Subject Classification (1985 Revision). Primary $40 \mathrm{H} 05$.

Key words and phrases. Sliding hump technique, BK-spaces, spaces with the Wilansky property. 
[S] make use of the sectional convergence in $E$ (when $E \subset c_{0}$ ), and the latter is no longer available (when $E \subset l^{\infty}$ ).

We obtain new classes of $B K$-spaces having the Wilansky property. For instance, we prove that every $B K$ - $A K$-space $E$, such that $S\left(E^{\prime}\right)$ is separably complemented in $E^{\prime}$, has the Wilansky property. Here $S\left(E^{\prime}\right)$ denotes the space of all $y \in E^{\prime}$ which have sectional convergence with respect to the norm.

We prove that the bidual $E^{\prime \prime}$ of a solid $B K-A K$-space $E$ whose dual $E^{\prime}$ is as well $B K-A K$ has the following separable Wilansky type property: If $D$ is a norm dense subspace of $E^{\prime \prime}$ containing $\Phi$ and having $D^{\beta}=E^{\beta} \quad\left(=E^{\prime}\right)$, then every separable $F K$-space $F$ containing $D$ must actually contain $E^{\prime \prime}$. When applied to the case $E=c_{0}$, this provides a result of Bennett and Kalton [BK ${ }_{1}$, W, p. 259].

Notation. The sections of a sequence $x \in \omega$ are denoted by

$$
P_{n} x=\sum_{i=1}^{n} x_{i} e^{i}
$$

where $e^{i}$ are the unit vectors. If $P_{n} x \rightarrow x \quad(n \rightarrow \infty)$, then $x$ is said to have sectional convergence. If $E$ is a $B K$-space, then $S(E)$ denotes the space of all $x \in E$ having sectional convergence with respect to the norm on $E$.

Concerning all other notions from sequence space theory, we refer to the book [W].

\section{THE MAIN THEOREM}

This section presents our fundamental result.

Theorem 1. Let $E$ be a $B K$ - $A K$-space such that $S\left(E^{\prime}\right)$ is complemented in $E^{\prime}$ with separable complement $L$. Let $M=S\left(E^{\prime}\right)^{\perp}$ be the annihilator of $S\left(E^{\prime}\right)$ in $E^{\prime \prime}$. Let $F$ be any subspace of $E^{\beta \beta}$ containing $\Phi$ and suppose $F^{\beta}=E^{\beta}$ $\left(=E^{\prime}\right)$. Then $\sigma\left(E^{\prime}, F+M\right)$ and $\sigma\left(E^{\prime}, \bar{F}+M\right)$ have the same convergent sequences.

Proof. We need some preparations. We may assume that $E$ has a monotone norm (see [W, p. 104]). Let $p_{1}: E^{\prime} \rightarrow S\left(E^{\prime}\right), p_{2}: E^{\prime} \rightarrow L$ be the projection operators corresponding with the decomposition $E^{\prime}=S\left(E^{\prime}\right) \oplus L$. Notice that $E^{\prime \prime}=S\left(E^{\prime}\right)^{\perp} \oplus L^{\perp}, L^{\prime}=S\left(E^{\prime}\right)^{\perp}=M$. We define norm continuous linear operators $Q_{n}: E^{\prime} \rightarrow E^{\prime}, n \in \mathbf{N}$, by

$$
Q_{n} y=P_{n} \circ p_{1} y+p_{2} y
$$

Then we have

$$
\left\|y-Q_{n} y\right\|=\left\|p_{1} y-P_{n} \circ p_{1} y\right\| \rightarrow 0 .
$$

We have to prove that $\sigma\left(E^{\prime}, F+M\right)$-convergent sequences are $\sigma\left(E^{\prime}, \bar{F}+M\right)$ convergent. To this end, it suffices to prove that every $\sigma\left(E^{\prime}, F+M\right)$-null 
sequence is bounded in norm. Indeed, suppose this has been proved for a $\sigma\left(E^{\prime}, F+M\right)$-null sequence $\left(y^{n}\right),\left\|y^{n}\right\| \leq K$, say. Then, for $\bar{x} \in \bar{F}$ fixed and $\varepsilon>0$ choose $x \in F$ having $\|x-\bar{x}\|<\varepsilon / K$. Then

$$
\left|\left\langle\bar{x}, y^{n}\right\rangle\right| \leq K\|x-\bar{x}\|+\left|\left\langle x, y^{n}\right\rangle\right|<\varepsilon
$$

for $n \geq n(\varepsilon)$.

Let $\left(y^{n}\right)$ be a $\sigma\left(E^{\prime}, F+M\right)$-null sequence and assume it is not bounded in norm, $\left\|y^{n}\right\| \geq n 2^{n}$, say. Let $v^{n}=y^{n} / n$.

I. There exist strictly increasing sequences $\left(k_{j}\right),\left(n_{j}\right)$ of integers such that the following conditions (1) and (2) are satisfied:

(1) $\left\|Q_{k_{j-1}} v^{n_{j}}\right\| \leq 2^{-j}, \quad j=1,2, \ldots$,

(2) $\left\|v^{n_{j}}-Q_{k_{j}} v^{n_{j}}\right\| \leq 2^{-j}, \quad j=1,2, \ldots$.

Suppose $k_{1}, \ldots, k_{j}$ and $n_{1}, \ldots, n_{j}$ have already been defined in accordance with (1) and (2). We claim that $\left\|Q_{k_{j}} v^{n}\right\| \rightarrow 0 \quad(n \rightarrow \infty)$. Since $\left(y^{n}\right)$ is $\sigma\left(E^{\prime}, F+M\right)$-null, $\left(p_{2} y^{n}\right)$ is bounded for $\sigma(L, M)$, hence is norm bounded, hence $\left\|p_{2} v^{n}\right\| \rightarrow 0$. On the other hand, $y^{n}=p_{1} y^{n}+p_{2} y^{n}$ implies that $\left(p_{1} y^{n}\right)$ is $\sigma\left(E^{\prime}, F+M\right)$-bounded, hence $\left(p_{1} v^{n}\right)$ is $\sigma\left(E^{\prime}, F+M\right)$-null, hence is coordinatewise null in view of $\Phi \subset F$. Clearly this implies $\left\|P_{k_{j}} p_{1} v^{n}\right\| \rightarrow 0$, proving our claim. But now it is clear that a choice of $n_{j+1}>n_{j}$ satisfying (1) is possible.

Next observe that $\left\|v^{n_{j+1}}-Q_{k} v^{n_{j+1}}\right\| \rightarrow 0 \quad(k \rightarrow \infty)$. This permits a choice of $k_{j+1}>k_{j}$ in accordance with (2).

II. Let $z^{j}=Q_{k_{j}} v^{n_{j}}-Q_{k_{j-1}} v^{n_{j}}=P_{k_{j}} p_{1} v^{n_{j}}-P_{k_{j-1}} p_{1} v^{n_{j}}$, and let $\alpha_{j}=1 /\left\|z^{j}\right\|$. Then $\left(\alpha_{j}\right)$ is an $l^{1}$-sequence by (1), (2). Observe that $\alpha_{j} z^{j} \rightarrow 0$ with respect to $\sigma\left(E^{\prime}, F+M\right)$, but $\left\|\alpha_{j} z^{j}\right\|=1$. Therefore, a result of Pelczyński [P] guarantees the existence of a basic subsequence $\left(\alpha_{j_{r}} z^{j_{r}}\right)$ of $\left(\alpha_{j} z^{j}\right)$. To simplify the reasoning in the following, we assume that $\left(\alpha_{j} z^{j}\right)$ itself is a basic sequence in $E^{\prime}$.

III. We claim the existence of a null sequence $\left(\lambda_{j}\right)$ such that the sequence $z$, defined by

$$
z_{k}=\lambda_{j} \alpha_{j} z_{k}^{j} \text { for } k_{j-1}<k \leq k_{j},
$$

is not an element of $S\left(E^{\prime}\right)$.

Let $G$ denote the subspace of $E^{\prime}$ consisting of all sequences

$$
z=\sum_{j=1}^{\infty} \lambda_{j} \alpha_{j} z^{j}
$$


where $\left(\lambda_{j}\right)$ is in $c_{0}$ and the series converges in norm. Define a linear operator $\varphi: G \rightarrow c_{0}$ by setting

$$
\varphi(z)=\varphi\left(\sum_{j=1}^{\infty} \lambda_{j} \alpha_{j} z^{j}\right)=\left(\lambda_{j}\right) .
$$

$\varphi$ is well defined since $\left(\alpha_{j} z^{j}\right)$ is a basic sequence by assumption. We prove that $\varphi$ is continuous. Let $z \in G, z=\sum \lambda_{j} \alpha_{j} z^{j}$. Then

$$
\begin{aligned}
\left|\lambda_{j}\right|=\left\|\lambda_{j} \alpha_{j} z^{j}\right\| & =\left\|\sum_{i=1}^{j} \lambda_{i} \alpha_{i} z^{i}-\sum_{i=1}^{j-1} \lambda_{i} \alpha_{i} z^{i}\right\| \\
& =\left\|P_{k_{j}} z-P_{k_{j-1}} z\right\| \leq 2\|z\|,
\end{aligned}
$$

the latter in view of the monotonicity of the norm on $E$ (and thus on $E^{\prime}$ ). This proves that $\varphi$ is continuous.

Let $\bar{G}$ be the norm closure of $G$ in $E^{\prime}$. Then $\varphi$ extends to a continuous, linear operator $\bar{\varphi}: \bar{G} \rightarrow c_{0}$. Let $z \in \bar{G}$, then $z=\sum \lambda_{j} \alpha_{j} z^{j}$ for some sequence $\left(\lambda_{j}\right)$, since $\left(\alpha_{j} z^{j}\right)$ is a basic sequence. But notice that $\bar{\varphi}(z)=\left(\lambda_{j}\right)$ by a $K$-space argument. So actually $\left(\lambda_{j}\right)$ is in $c_{0}$, hence $z \in G$, proving $G=\bar{G}$.

Notice that $\varphi$ is a continuous injection. This proves that $\varphi$ is not surjective. For supposing it were, it would be a homeomorphism by the open mapping theorem, i.e. we would have $G \approx c_{0}$. But this is absurd, since no separable dual space may contain a copy of $c_{0}$. So $\varphi$ is not surjective. Let $\left(\lambda_{j}\right)$ be any null sequence which is not in the range of $\varphi$. We prove that $z$, defined by $(*)$, is not in $S\left(E^{\prime}\right)$. Indeed, $z \in S\left(E^{\prime}\right)$ would imply $\left\|z-P_{k_{j}} z\right\| \rightarrow 0 \quad(j \rightarrow \infty)$. But note that

$$
P_{k_{j}} z=\sum_{i=1}^{j} \lambda_{i} \alpha_{i} z^{i},
$$

hence $z$ would be in $G$, which was excluded. This ends step III.

IV. We prove that $\left(P_{k} z\right)$ is $\sigma\left(E^{\prime}, F+M\right)$-convergent with limit $z$. Indeed, let $x \in F+M, k \in \mathbf{N}, k_{j-1}<k \leq k_{j}$. Then we have

$$
\left\langle x, P_{k} z\right\rangle=\sum_{i=1}^{j-1} \lambda_{i} \alpha_{i}\left\langle x, z^{i}\right\rangle+\lambda_{j} \alpha_{j}\left\langle x, P_{k} z^{j}\right\rangle .
$$

Here the first summand converges $\left(k \rightarrow \infty, k_{j-1}<k \leq k_{j}\right)$ since $\left\langle x, z^{i}\right\rangle \rightarrow 0$ and $\left(\alpha_{j}\right) \in l^{1}$. But the second summand converges as well in view of $\lambda_{j} \rightarrow 0$ $\left(k \rightarrow \infty, k_{j-1}<k \leq k_{j}\right)$ and

$$
\left|\alpha_{j}\left\langle x, P_{k} z^{j}\right\rangle\right|=\left|\left\langle P_{k} x, \alpha_{j} z^{j}\right\rangle\right| \leq\left\|P_{k} x\right\|\left\|\alpha_{j} z^{j}\right\| \leq\|x\| .
$$

In view of $F^{\beta}=E^{\prime}$ this implies $z \in E^{\prime}$ and so $P_{k} z \rightarrow z$ in $\sigma\left(E^{\prime}, F+M\right)$. 
Now observe that the operators $Q_{r}$ are $\sigma\left(E^{\prime}, F+M\right)$-continuous, so $Q_{r}\left(P_{k} z\right) \rightarrow$ $Q_{r} z \quad(k \rightarrow \infty)$, proving $P_{r} z=Q_{r} z$, hence $z \in S\left(E^{\prime}\right)$. But this contradicts step III and therefore ends the proof.

In the case where $S\left(E^{\prime}\right)=E^{\prime}$, i.e. when $E^{\prime}$ has sectional convergence, the proof may be simplified. Here we have $M=\{0\}, Q_{n}=P_{n}$. This yields the following.

Corollary 1. Let $E$ be a $B K-A K$-space such that $E^{\prime}$ is as well $B K-A K$. Let $F$ be a subspace of $E^{\prime \prime}$ containing $\Phi$ and satisfying $F^{\beta}=E^{\beta}\left(=E^{\prime}\right)$. Then $\sigma\left(E^{\prime}, F\right)$ and $\sigma\left(E^{\prime}, \bar{F}\right)$ have the same convergent sequences.

\section{SPACES WITH THE WILANSKY PROPERTY}

An $F K$-space $E$ is said to have the Wilansky property if every subspace $F$ of $E$ satisfying $F^{\beta}=E^{\beta}$ is barrelled in $E$ (see $\left[\mathrm{B}_{2}\right]$ ). In $\left[\mathrm{B}_{2}\right]$ and $[\mathrm{S}]$ it is proved that every $B K$ - $A K$-space $E$ whose dual $E^{\prime}$ is as well a $B K-A K$-space has the Wilansky property. Here we obtain:

Theorem 2. Let $E$ be a $B K$ - $A K$-space such that $S\left(E^{\prime}\right)$ has a separable complement $L$ in $E^{\prime}$. Let $G$ be any $F K$-space having $E \subset G \subset E^{\beta \beta}$. Then $G$ has the Wilansky property if and only if $E$ is of finite codimension in $G$.

Proof. Necessity. Suppose $E$ is of infinite codimension in $G$. Let $\left(y^{n}\right)$ be a linearly independent sequence in $G \backslash E$. Since $E^{\beta}=G^{\beta}, E$ is barrelled as a subspace of $G$, hence is closed in $G$. But now $F=E+\operatorname{lin}\left\{y^{n}: n \in \mathbf{N}\right\}$ is a subspace of $G$ having $F^{\beta}=G^{\beta}$ which is not barrelled. Indeed, we may define a sequence $\left(f_{n}\right)$ in $G^{\prime}$ such that $f_{n}$ is 0 on $E+\operatorname{lin}\left\{y^{1}, \ldots, y^{n-1}\right\}$ and satisfies $f_{n}\left(y^{n}\right)=n\left|y^{n}\right|$ (for some continuous seminorm $|\cdot|$ on $G$ ). Then $f_{n} \rightarrow 0$, $\sigma\left(G^{\prime}, F\right)$, but $\left(f_{n}\right)$ is not bounded in $G^{\prime}$.

Sufficiency. Let $F$ be a subspace of $G$ with $F^{\beta}=G^{\beta}$. We may assume that $F$ contains $\Phi$ (see $\left[\mathrm{B}_{2}\right.$, Theorem 1$]$ ).

Let $U$ be a barrel in $F$. Since $M \cap E=\{0\}, M=S\left(E^{\prime}\right)^{\perp}$, the space $F \cap M$ is finite dimensional. Let $S$ be some topological complement of $F \cap M$ in $M$. Let $B$ denote the unit ball in $S$. Note that $B$ is $\sigma\left(E^{\prime \prime}, E^{\prime}\right)$-compact, since the unit ball in $M \approx L^{\prime}$ is weak * compact and $S$ is of finite codimension in $M$. Now let $V=U+B$. Then $V^{0}$, the $\left\langle E^{\prime \prime}, E^{\prime}\right\rangle$-polar of $V$, is $\sigma\left(E^{\prime}, F+M\right)$ bounded, since $V$ spans $F+M$. By Theorem 1, $\sigma\left(E^{\prime}, F+M\right)$-bounded sets are norm bounded in $E^{\prime}$, so that $V^{0}$ is norm bounded in $E^{\prime}$. Hence $V^{00}$ is a norm neighbourhood of 0 in $E^{\prime \prime}$, hence $V^{00} \cap F$ is a norm neighbourhood of 0 in $F$, since $G$ (and hence $F$ ) must have the topology induced by $E^{\prime \prime}$. We end the proof by showing $V^{00} \cap F \subset U$. By the definition of $V$, we have $V^{00}=\bar{U}+B$, the closure being taken in $\sigma\left(E^{\prime \prime}, E^{\prime}\right)$, since $B$ is $\sigma\left(E^{\prime \prime}, E^{\prime}\right)$ compact. But $V^{00} \cap F=\bar{U} \cap F$ in view of $B \cap F=\{0\}$. Since $F$ has only finitely many dimensions "outside $E$ ", we deduce that $\bar{U} \cap F=U$, which ends the proof of Theorem 2 . 
Corollary 2. [ $\mathrm{B}_{2}, \S 6$ ]. $c$ and $c s$ have the Wilansky property.

More generally, a $B K-A K$-space $E$ has the Wilansky property if $S\left(E^{\prime}\right)$ is of finite codimension in $E^{\prime}$, and the same is true for any $G$ having $E \subset G \subset E^{\beta \beta}$ such that $E$ is of finite codimension in $G$. In a forthcoming paper [NS], we use this fact to prove that for every invertible permanent triangular matrix $A$ whose inverse $A^{-1}$ is a bidiagonal matrix, the convergence domain $c_{A}$ has the Wilansky property.

Remark. In Theorems 1,2, the assumption that $E$ has separable dual may be replaced by any condition ensuring that $c_{0}$ does not embed into $E^{\prime}$. See for instance $[\mathrm{Kw}]$.

\section{SEPARABLE WiLANSKY PROPERTY}

It is clear from Theorem 2 that the bidual $E^{\prime \prime}$ of a $B K-A K$-space $E$ whose dual $E^{\prime}$ is as well $B K-A K$ does not have the Wilansky property unless $E$ has finite codimension in $E^{\prime \prime}$. Nevertheless, the bidual space $E^{\prime \prime}$ satisfies some weaker Wilansky type property, which might be called the separable Wilansky property.

Theorem 3. Let $E$ be a solid $B K-A K$-space whose dual $E^{\prime}$ is as well $B K-A K$. Let $D$ be a norm dense subspace of $E^{\prime \prime}$ containing $\Phi$ and satisfying $D^{\beta}=$ $E^{\beta} \quad\left(=E^{\prime}\right)$. Then every separable $F K$-space $F$ which contains $D$, actually contains $E^{\prime \prime}$.

Proof. Let $x \in E^{\prime \prime}$ be fixed. Since $D$ is a norm dense in $E^{\prime \prime}$, it is also $\tau\left(E^{\prime \prime}, E^{\prime}\right)$-sequentially dense in $E^{\prime \prime}$, i.e. there exists a sequence $\left(x^{n}\right)$ in $D$ which converges to $x$ in $\tau\left(E^{\prime \prime}, E^{\prime}\right)$. We claim that $\tau\left(E^{\prime \prime}, E^{\prime}\right) \mid D=\tau\left(D, E^{\prime}\right)$.

Indeed, by Theorem $1, \sigma\left(E^{\prime}, D\right)$ and $\sigma\left(E^{\prime}, E^{\prime \prime}\right)$ have the same convergent sequences, hence the same compact sets [W, p. 252]. This implies $\tau\left(E^{\prime \prime}, E^{\prime}\right) \mid D$ $=\tau\left(D, E^{\prime}\right)$.

Consequently, the sequence $\left(x^{n}\right)$ is Cauchy in $\left(D, \tau\left(D, E^{\prime}\right)\right)$. We prove that the inclusion mapping $i:\left(D, \tau\left(D, E^{\prime}\right)\right) \rightarrow F$ is continuous. This is a consequence of Kalton's closed graph theorem (see [ $\mathrm{BK}_{2}$, Theorem 5]), for $\sigma\left(E^{\prime}, D\right)$ is sequentially complete. Indeed, since $\sigma\left(E^{\prime}, D\right)$ and $\sigma\left(E^{\prime}, E^{\prime \prime}\right)$ have the same convergent sequences, they also have the same Cauchy sequences. But $\sigma\left(E^{\prime}, E^{\prime \prime}\right)$ is sequentially complete as a consequence of the fact that $E$, and hence $E^{\prime}=E^{\alpha}$, is solid. This proves that $\sigma\left(E^{\prime}, D\right)$ is sequentially complete.

Since $i:\left(D, \tau\left(D, D^{\beta}\right)\right) \rightarrow F$ is continuous, the sequence $\left(x^{n}\right)$ is Cauchy in $F$, and hence converges to some $\bar{x} \in F$. From $K$-space reasons, we have $x=\bar{x}$, proving $x \in F$.

Certainly, in Theorem 3 , the solidity of the space $E$ may be replaced by the condition that $\sigma\left(E^{\prime}, E^{\prime \prime}\right)$ is sequentially complete.

Corollary 3. (Compare $\left[\mathrm{BK}_{1}\right.$, Theorem 3].) Let $F$ be a separable $F K$-space containing $\Phi$ and suppose $F \cap l^{\infty}$ is norm dense in $l^{\infty}$. Then $l^{\infty} \subset F$. 
Proof. This follows from Theorem 3 and the fact that every norm dense subspace $D$ of $l^{\infty}$ satisfies $D^{\beta}=l^{1}$ (see [W, Lemma 16.3.3]).

The result of Bennett and Kalton has been generalized by Snyder [Sn] to a nonseparable version. He proves that every $F K$-space $F$ containing $\Phi$ and satisfying $F+c_{0}=l^{\infty}$ must have $F=l^{\infty}$.

\section{SCARCE COPIES}

The concept of scarce copies of sequence spaces has been introduced by Bennett $\left[\mathrm{B}_{1}\right]$. He proves that every scarce copy of $\omega$ and $l^{1}$ is barrelled, but that all other standard sequence spaces do not have this property. For instance, $c_{0}$ does not have any barrelled scarce copy at all (see $\left[\mathrm{B}_{1}\right]$ for details). Here we obtain another negative result on the barrelledness of scarce copies.

Theorem 4. Let $E$ be a $F K$-AB-space contained in $l^{\infty}$ such that $E^{\gamma} \subset b s$. Then $E$ does not have any barrelled scarce copy.

Proof. Suppose $\Sigma(E, r)$ is a barrelled scarce copy of $E$. This implies $\Sigma(E, r)^{\beta}$ $\subset E^{f}=E^{\gamma}$, the latter since $E$ has $A B$ (see [W, p. 167]). Therefore $\Sigma(E, r)^{\beta}$ $\subset b s$.

We prove that $\Sigma\left(c_{0}, r\right)$ is a barrelled scarce copy of $c_{0}$, thus obtaining a contradiction, since $c_{0}$ has no barrelled scarce copies. Since $c_{0}$ has the Wilansky property, barrelledness of $\Sigma\left(c_{0}, r\right)$ will be a consequence of $\Sigma\left(c_{0}, r\right)^{\beta} \subset l^{1}$. So let $y \notin l^{1}$. Since $c_{0}^{\gamma}=l^{1}$, there exists $x \in c_{0}$ such that $x y \notin b s$, hence $x y \notin \Sigma(E, r)^{\beta}$. Let $z \in \Sigma(E, r)$ be chosen with $x y z \notin c s$. By the definition of $\Sigma(E, r)$, there exist $z^{1}, \ldots, z^{n} \in \sigma(E, r)$ having $z=z^{1}+\cdots+z^{n}$. This implies $x y z^{i} \notin c s$ for some $i$. We claim that $x z^{i} \in \sigma\left(c_{0}, r\right) \subset \Sigma\left(c_{0}, r\right)$. Since $z^{i} \in E \subset l^{\infty}$, we have $x z^{i} \in c_{0}$. On the other hand,

$$
c_{n}\left(x z^{i}\right) \leq c_{n}\left(z^{i}\right) \leq r_{n}
$$

for every $n$ implies $x z^{i} \in \sigma\left(c_{0}, r\right)$. This proves $y \notin \Sigma\left(c_{0}, r\right)^{\beta}$.

\section{REFERENCES}

[ $B_{1}$ ] G. Bennett, Some inclusion theorems for sequence spaces, Pacific J. Math. 46 (1973), 17-30.

[ $\mathbf{B}_{2}$ ] _ - Sequence spaces with small $\beta$-duals, Math. Z. 194 (1987), 321-329.

[ $\mathbf{B K}_{1}$ ] G. Bennett and N. J. Kalton, Addendum to " $F K$-spaces containing $c_{0}$ ", Duke Math. J. 39 (1972), 819-821.

[ $\mathbf{B K}_{2}$ ] _ _ Inclusion theorems for $K$-spaces, Canad. J.Math. 25 (1973), 511-524.

[ Kw] S. Kwapien, On Banach spaces containing $c_{0}$, Studia Math. 52 (1974), 187-188.

[ NS ] D. Noll and W. Stadler, Zerlegung von Wachstumsbereichen und Wirkfeldern für die Verfahren bewichteter Mittel, Manuscripta Math. 60 (1988), 197-209.

[P] A. Pelczyński, A note on the paper of I. Singer: "Basic sequences and reflexivity in Banach spaces", Studia Math. 21 (1962), 371-374.

[ Sn ] A. K. Snyder, A property of the embedding of $c_{0}$ in $l_{\infty}$, Proc. Amer. Math. Soc. 97 (1986), 59-60. 
[S ] W. Stadler, Zu einer Frage von Wilansky, Arch. Math. 48 (1987), 149-152.

[ W] A. Wilansky, Summability through functional analysis, North-Holland, New York, 1984.

Universität Stuttgart, Mathematisches Institut B, Pfaffenwaldring, 7000 STUTTGART 80, BRD

Universität Stuttgart, Mathematisches Institut A, Pfaffenwaldring, 7000 STUTTGART 80, BRD 